\title{
Full Field Imaging Ellipsometry (FFIE) Platform Using CCD Camera and Advanced Software for Simultaneous Spots' Sensing and Measurement
}

Avi Karsenty, Lev Academic Center, Faculty of Engineering, Department of Applied Physics/ElectroOptics Engineering, Jerusalem, Israel

Shmuel Feldman, Lev Academic Center, Faculty of Engineering, Department of Applied Physics/ Electro-Optics Engineering, Jerusalem, Israel

Zvi Veig, Lev Academic Center, Faculty of Engineering, Department of Applied Physics/ElectroOptics Engineering, Jerusalem, Israel

Yoel Arieli, Lev Academic Center, Faculty of Engineering, Department of Applied Physics/ElectroOptics Engineering, Jerusalem, Israel

\section{ABSTRACT}

This article describes a new approach for performing full field imaging ellipsometry. In this new technique, the objective lens of a high numerical aperture microscope is used to illuminate the surface of a $2 \mathrm{D}$ object. The light reflected from each point of the surface is gathered by the same lens and projected onto a 2D CCD detectors array; thus, enabling the measurement of numerous surface points simultaneously. Using this simple method, areas of up to $0.9 \mathrm{~cm}^{2}$ can be measured with high accuracy. Since the nanotechnology domain is rapidly growing, such a technique can bring benefits to the scientific community, facing the need to analyze large surfaces of thin films.

\section{KEYWORDS}

CCD, Ellipsometry, Large Surfaces, Sensing

\section{INTRODUCTION}

Ellipsometry is a well-known Non-Destructive Evaluation (NDE) optical method for determining film thickness and optical properties. It measures the change in the state of polarization of light reflected from films' surface. Fast ellipsometry methods, single or multi-wavelengths, have been adopted for monitoring film growth in situ, allowing 
for the precise control of film deposition processes (Muller, 2005). With time, several parameters have been separately and largely investigated, such as in-situ measurements of materials complex permittivity using reflection ellipsometry (Sagnard, 2005), and arbitrary refractive index profile of composite thin films (Ho, 1990).

The advancement of spectroscopic ellipsometers has extended the analytical power of ellipsometry to complex multilayer coatings, where several optical parameters (refractive index, extinction coefficient, film thickness, roughness anisotropy, etc.) can be determined simultaneously. In the last two decades, new generations of ellipsometers came up with additional specifications and techniques, such as spectroscopic ellipsometry (Jung, 2008), methods of calibration (Asinovski, 2008), stroboscopic illumination technique (Han, 2006), and customization (Acurion, 2004). Even apertureless optical near-field scanning microscope system was created by combining a commercially available atomic force microscope and an Ellipsometer (Karageorgiev, 2001). Moreover, ellipsometry became suitable for optical sensing (Arwin, 2001) and film sensing applications (Sinibaldi, 2013).

A big leap ahead was stepped forward by developing new techniques of imaging ellipsometry (IE), e.g. combined microscopy and Thin Film Characterization (TFC), which overcomes the limits of classical ellipsometry, the spatial resolution. Besides the determination of film thickness and optical properties, one receives high contrast ellipsometric images from the surface with highest resolution. Small samples, structured materials or inhomogeneous surfaces, which are beyond the capabilities of other ellipsometric tools, are now visible with IE and this opens new doors to a better understanding of today's advanced thin film and biological applications. However, the known IE are still complicated or limited in their performance. For example, the moving ellipsometry system based on Polarizer Compensator Surface Analyzer (PCSA) components, and enabling a null ellipsometry technique, is not strong enough to measure large surfaces.

The conventional technique mentioned above enables several points of measurement, since the mechanical rotor enables a scan along the surface. The Imaging Ellipsometer overcomes this limitation by using a motorized focusing mechanism to collect a series of images with different foci within the field-ofview. The problem is that in such a system, the measurement time is long, since in each point there is a rotation of the polarizers. Also, the mechanical motor may require accuracy, and as such, may involve high cost. Similar concerns of cost and complexity can be cited for a multifocal ellipsometer system. Thus, there is a need for an IE which is more user friendly, cheap and sufficiently accurate for surface measurements. In the past, several biosensors based on imaging ellipsometry have been developed, mainly for bio-medical applications, such as bio-sensors in the long study works of Jin (1995, 2008, and 2011), and of Wang (2004). 


\section{RESULTS AND DISCUSSION}

\subsection{Presentation of the FFIE New Approach}

In this article, an extended new approach (Arieli, 2012) is presented for performing Full Field IE (FFIE) over large surfaces, as schematically shown in Figure 1. This new approach for imaging ellipsometry enables the performance of simultaneous $2 \mathrm{D}$ measurements of a thin layered object and to integrate all the mapped spots in order to obtain a full 2D ellipsometric measurement of the object.

The system is based on a coherent point light source emitting a diverging beam. A microscope's objective lens with high numerical aperture is used to illuminate an object's surface. The light reflected from each point of the surface is gathered by the same lens and projected onto a CCD detector. As described in Figure 1, each point of the surface is illuminated by a light ray with a different angle relative to the optical axis, and is imaged by the objective lens on a different pixel of the CCD detector. Thus, each pixel in the detectors array represents a different point on the surface of the object. In this way, numerous surface points may be measured simultaneously.

However, there is one main disadvantage when using this method; there are some points on the object's surface that are illuminated by rays with relatively small angles of incidence, when compared to an ordinary Ellipsometer $\left(\sim 72^{\circ}\right)$. This fact can decrease

Figure 1. Schematic description of the FFIE proposed system

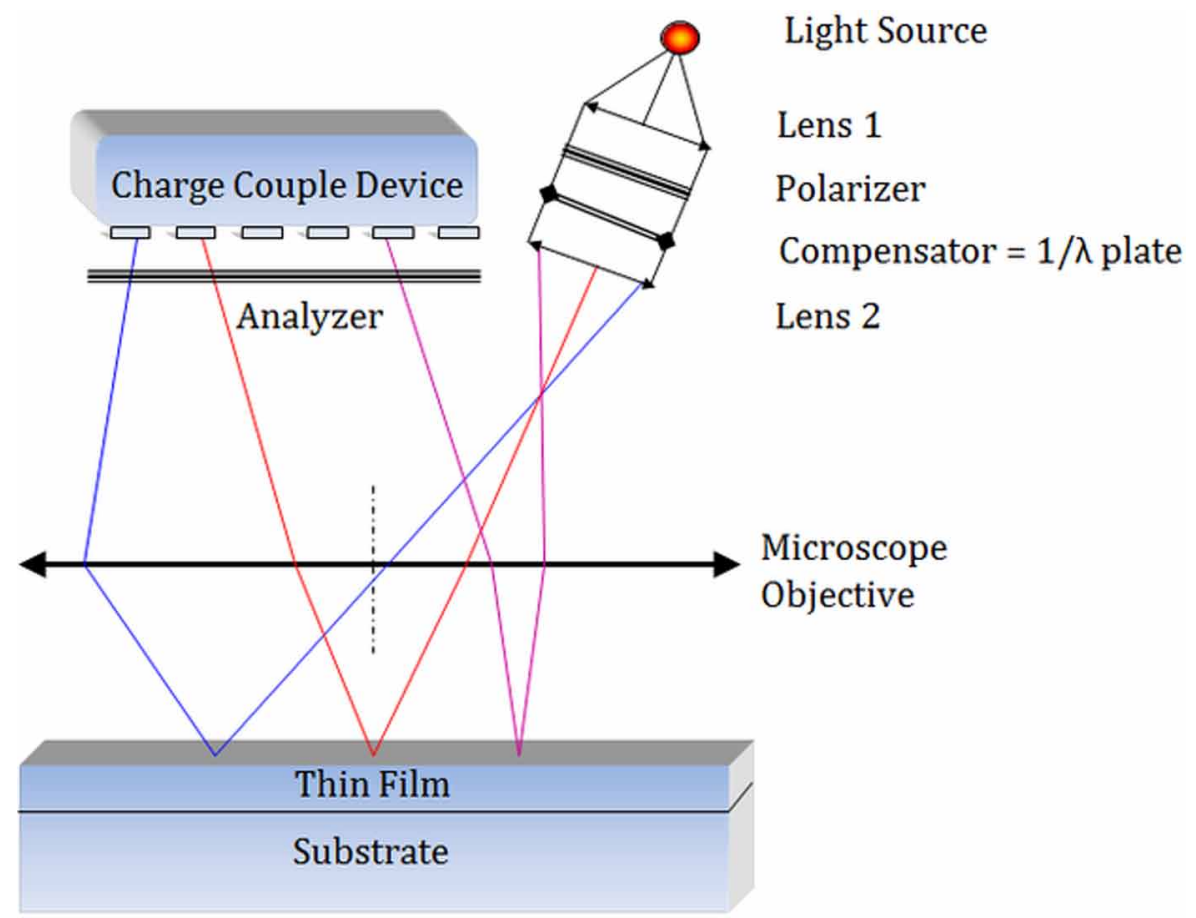


the resolution of the ellipsometric measurements at those points and limit the area that can be measured with sufficient accuracy.

In order to evaluate this approach we have designed the optical system and simulated the whole process.

\subsection{The Ellipsometric Measurement Simulation}

The first step to evaluate the performance of the suggested imaging ellipsometry method was to define the resolution of the measurement as a function of the angle of illumination. We have defined the measurement resolution $r$ as the rate of change of the intensity on the detector as a function of the thickness change:

$$
r=\frac{\Delta I}{\Delta D_{\text {film }}}
$$

where $\Delta I$ is the change in light intensity obtained on the detector, and $\Delta D_{\text {film }}$ the change in the thickness of the measured surface.

This approach is more detection-related than from ellipsometry itself. Since there are different types of Ellipsometers, each one sharing its own sensitivity, and assuming a well-defined tool/Ellipsometer, we present a generic approach w/o calculating the SNR.

The second step was to develop advanced Matlab software script, in order to simulate the imaging ellipsometer measurements through the different elements of the system, as presented in Figure 2. In the simulation, a light beam is propagated through the system, passing through a polarizer and a compensator, and meeting the upper measured surface in a certain angle of incidence. The reflected light beam is propagating through an analyzer towards the CCD.

\section{Figure 2. Schematics of the Ellipsometer proposed system's elements}

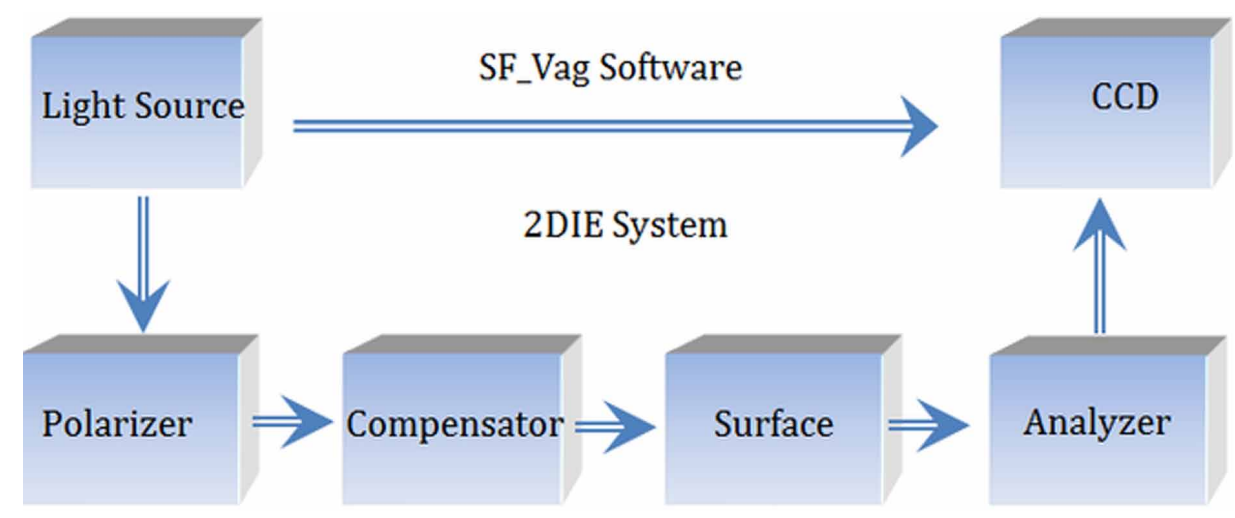


As the rotation angles of the polarizer and the analyzer vary, the light intensity in the CCD also varies and at certain rotation angles of the polarizer and the analyzer, the light intensity would be minimal, as usually done in null ellipsometry technique. By the intensity light function, as a function of the rotation angles of the polarizer and the analyzer, the optical properties of the object can be calculated. The characteristic matrix $J_{s}$ of the whole process was calculated by multiplying the characteristic matrices of all elements at the varying rotation angles, as presented in Equation 2:

$$
J_{s}=J_{p}(P) \cdot J_{\text {object }} \cdot J_{W P} \cdot J_{A}(A)
$$

where:

- $J_{P}$ is the characteristic matrix of the polarizer as a function of its rotation angle P;

- $J_{\text {object }}$ is the characteristic matrix of the object;

- $J_{W P}$ is the characteristic matrix of a fixed compensator;

- $J_{A}$ is the characteristic matrix of the analyzer as a function of its rotation angle A.

The fixed compensator was determined as a quarter wavelength plate positioned in an angle of $45^{\circ}$ when compared to the axis.

The characteristic matrix of an object consists of an infinite substrate coated by thin layers was calculated using the Thin Film Transfer Matrix method (Born, 1964). Thus, the light intensity in the CCD, as a function of the polarizer's and the analyzer's angles, is given by:

$$
\begin{aligned}
& I_{\text {Final }}(P, A)= \\
& 1 / \sqrt{2} \cdot\left(J_{S}^{1,1} \cdot E_{0 x}+J_{S}^{1,2} \cdot E_{0 y}\right)\left(J_{S}^{1,1} \cdot E_{0 x}+J_{S}^{1,2} \cdot E_{0 y}\right)^{*} \\
& +1 / \sqrt{2} \cdot\left(J_{S}^{2,1} \cdot E_{0 x}+J_{S}^{2,2} \cdot E_{0 y}\right)\left(J_{S}^{2,1} \cdot E_{0 x}+J_{S}^{2,2} \cdot E_{0 y}\right)^{*}
\end{aligned}
$$

where $E_{0 x}$ and $E_{0 y}$ are the amplitudes of the $\mathrm{X}$ and $\mathrm{Y}$ polarizations of the illumination light.

Using Equation 2 and Equation 3 the ellipsometric measurement's resolution of an object as a function of the illumination angle, were calculated. The object was assumed to be an infinite thickness substrate with refractive index $n_{0}=3.6$ coated by a layer of thickness $h=50 \AA$ and refractive index $n_{l}=1.45$.

\subsection{The 2D Imaging Ellipsometric Optical System}

In order to simulate the imaging process and the rays' incident angles on each point of the object, a typical imaging system was simulated using the Oslo 6 optical design 
software (Lambda, 2017). The light source was located off the optical axis such that its light rays illuminate the object obliquely. The lens used for the Imaging Ellipsometry part has a magnification of $x 60$ and a N.A. of 0.95 (Matsubara, 1977). The first criterion to find the measured surface spot is to identify the light beam entering the system and fully received by the CCD. The area is $250 \mu \mathrm{m} \times 250 \mu \mathrm{m}$ with high resolution, and the detector size is $1.5 \mathrm{~cm} \times 1.5 \mathrm{~cm}$. The resolution is $\sim 0.5 \mathrm{um}$ for above area, $1000 \mathrm{x}$ 1000 pixels.

As shown in Figure 3, part of the beam is not reflected by the optical system, and as a consequence is not useful.

The whole system was optimized such that the light ray will be fully received by the CCD, as shown in Figure 4.

Figure 3. Presentation of the Oslo pre-optimization stage of the FFIE system

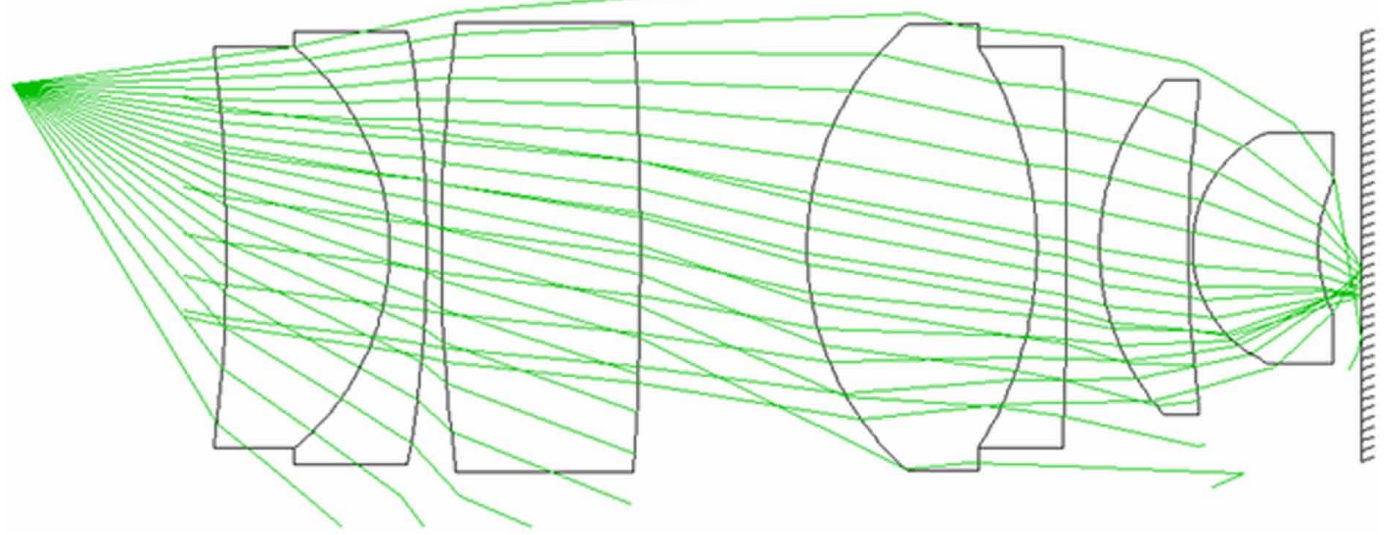

Figure 4. Presentation of the Oslo post-optimization stage of the FFIE system

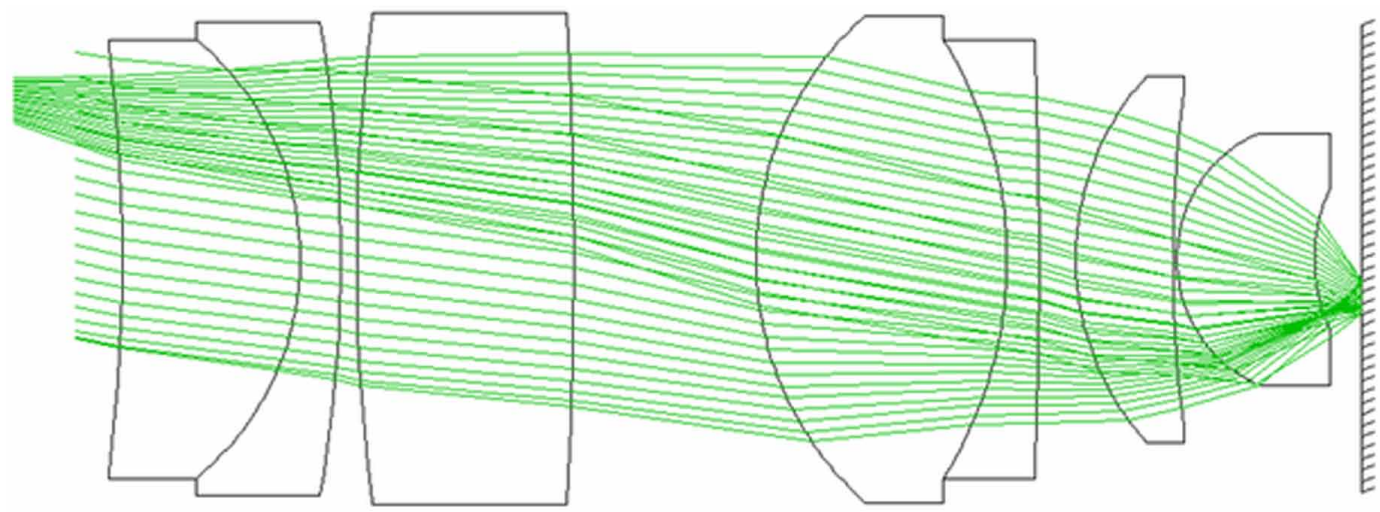


The lens was originally designed for direct imaging. The lens parameters have been inserted into Oslo software, and optimized for oblique imaging. Much effort was invested in Oslo in order to correct the original aberrations of the lens. Optimization was obtained by Oslo to correct the depth issues and aberrations.

The specifications of the different planes and lenses after optimization are presented in Figure 5. For example, it is interesting to pay attention that plane number 13 built from reflective material, serves as a measured layer inside the whole Ellipsometer system.

Since different points on the surface have a different incident angle, questions regarding the calibration and accuracy can rise. In order to address this concern, the calculation is performed in the following way: we start from the detector (its position is known). We trace reverse tracing from each detector to the object and calculate the corresponding angle of incidence. Since the aim of this microscope is not to enable imaging but imaging ellipsometry, then using reverse tracing, we definitely know how to calculate each camera's pixel footprint and measurement. So, we build accordingly the ellipsometric map of the object.

By the optical design software, each detector in the detectors array was imaged on the object's surface and the rays' incident angles from the light source on that spot on the object's surface was calculated. For each detector, the intensity of the reflected light was attained and the resolution was determined as described above. Figure 6 presents the results of the resolution as a function of the incidence angle. The results show that the resolution rapidly decreases as the incident angle deviates from the $\sim 72^{\circ}$ standard angle. As mentioned above, the angle of the rays was calculated according to the knowledge of the location of each pixel in the camera and the light source.

Figure 5. Specifications of the optimized FFIE system

\begin{tabular}{|c|c|c|c|c|c|}
\hline $\begin{array}{l}\text { SRF } \\
\text { OBJ }\end{array}$ & RADIUS & $\begin{array}{r}\text { THICKNESS } \\
100.000000\end{array}$ & $\begin{array}{l}\text { APERTURE RADIUS } \\
1.0000 \mathrm{e}-04\end{array}$ & $\begin{array}{r}\text { GLASS } \\
\text { AIR }\end{array}$ & $\begin{array}{c}\text { SPE } \\
=\end{array}$ \\
\hline $\begin{array}{l}\text { AST } \\
2 \\
3\end{array}$ & $\begin{array}{l}-753.114000 \\
-121.010000 \\
-577.791000\end{array}$ & $\begin{array}{r}76.280000 \\
17.373000 \\
6.889000\end{array}$ & $\begin{array}{r}92.000000 \mathrm{~A} \\
94.000000 \\
101.300000\end{array}$ & $\begin{array}{r}F K 51 \\
\text { PCD } 4 \\
\text { AIR }\end{array}$ & $\begin{array}{c}C \\
M\end{array}$ \\
\hline $\begin{array}{l}4 \\
5\end{array}$ & $\begin{array}{r}808.826000 \\
-1.6357 e+03\end{array}$ & $\begin{array}{l}93.153000 \\
77.878000\end{array}$ & $\begin{array}{l}103.700000 \\
105.300000\end{array}$ & $\begin{array}{r}\text { PCD } 4 \\
\text { AIR }\end{array}$ & $P$ \\
\hline $\begin{array}{l}6 \\
7 \\
8\end{array}$ & $\begin{array}{r}139.381000 \\
-175.224000 \\
-2.1297 e+03\end{array}$ & $\begin{array}{r}107.531000 \\
13.878000 \\
15.576000\end{array}$ & $\begin{array}{r}104.300000 \\
94.000000 \\
89.700000\end{array}$ & $\begin{array}{l}\text { CAF } \\
\text { SF3 } \\
\text { AIR }\end{array}$ & $\stackrel{c}{c}$ \\
\hline 19 & $\begin{array}{l}116.217000 \\
571.301000\end{array}$ & $\begin{array}{r}41.635000 \\
1.697000\end{array}$ & $\begin{array}{l}78.300000 \\
71.700000\end{array}$ & $\begin{array}{l}\text { CAF } \\
\text { AIR }\end{array}$ & $P$ \\
\hline $\begin{array}{l}11 \\
12\end{array}$ & $\begin{array}{l}59.007000 \\
70.289000\end{array}$ & $\begin{array}{l}58.907000 \\
20.000000\end{array}$ & $\begin{array}{l}54.000000 \\
30.000000\end{array}$ & $\begin{array}{r}\text { LAFN28 } \\
\text { AIR }\end{array}$ & $c$ \\
\hline 13 & -- & -20.000000 & 100.000000 & REFL_HATCH & \\
\hline
\end{tabular}


Figure 6. Result of the system measurement's resolution, as a function of the incident angle for a substrate coated by a film sharing a thickness of $\mathbf{5 0}$ angstroms. The graph shows that the resolution decreases as the incident angle deviates from the $72^{\circ}$ standard angle.

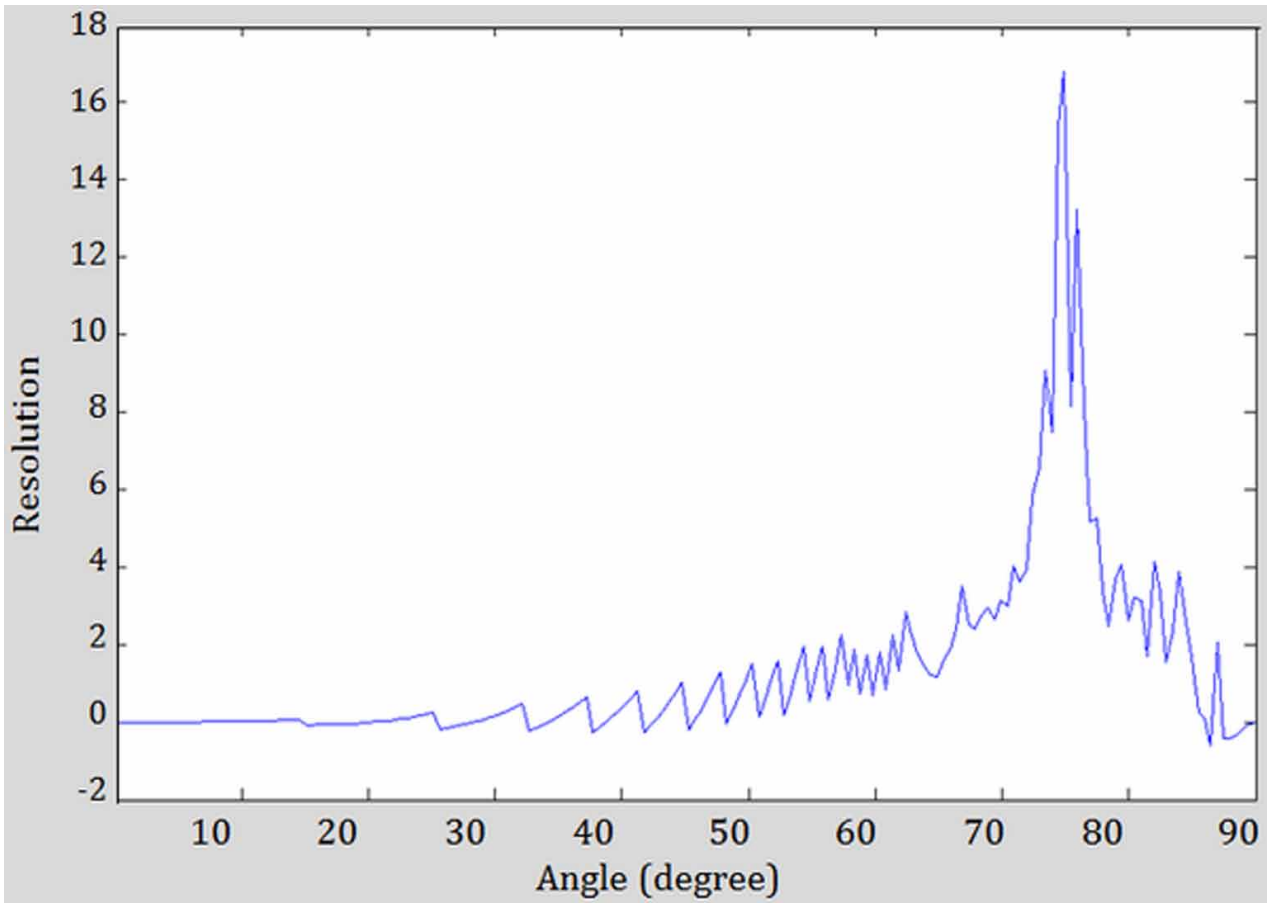

The resolution behavior as a function of the optical thickness of the coating layer. The results for two different incident angles, $72^{\circ}$ and $36^{\circ}$ are shown in Figure 7 . The results show that the resolution of the measurements is thickness dependent and is periodic as function of the thickness. The results also show that the resolution of the measurements at $36^{\circ}$ is smaller than the resolution of the measurements at $72^{\circ}$ by at least one order of magnitude at all thicknesses.

\subsection{Measured Surface and Relevant Angles}

The locus of the images of several detectors' lines (from the detectors array on the object's surface), are shown in Figure 8. Also, the rays' incident angles are shown on the object's surface, and which of them are related to each detector. According to the resolution results, we have limited the effective measured surface of the object only to that part which is having incident angles exceeding $36^{\circ}$, such that measurement resolution will still be acceptable. This limited part is shown by the dotted line in Figure 8. We have found that at least $0.45 \mathrm{~cm}^{2}$ of the surface is illuminated by rays with incident angles exceeding $36^{\circ}$, enabling good enough resolution. However, due to the symmetry, the overall effective measured surface 
Figure 7. Result of the system measurement solution, as a function of the coating layer's optical thickness for 2 compared angles, $72^{\circ}$ and $36^{\circ}$ system

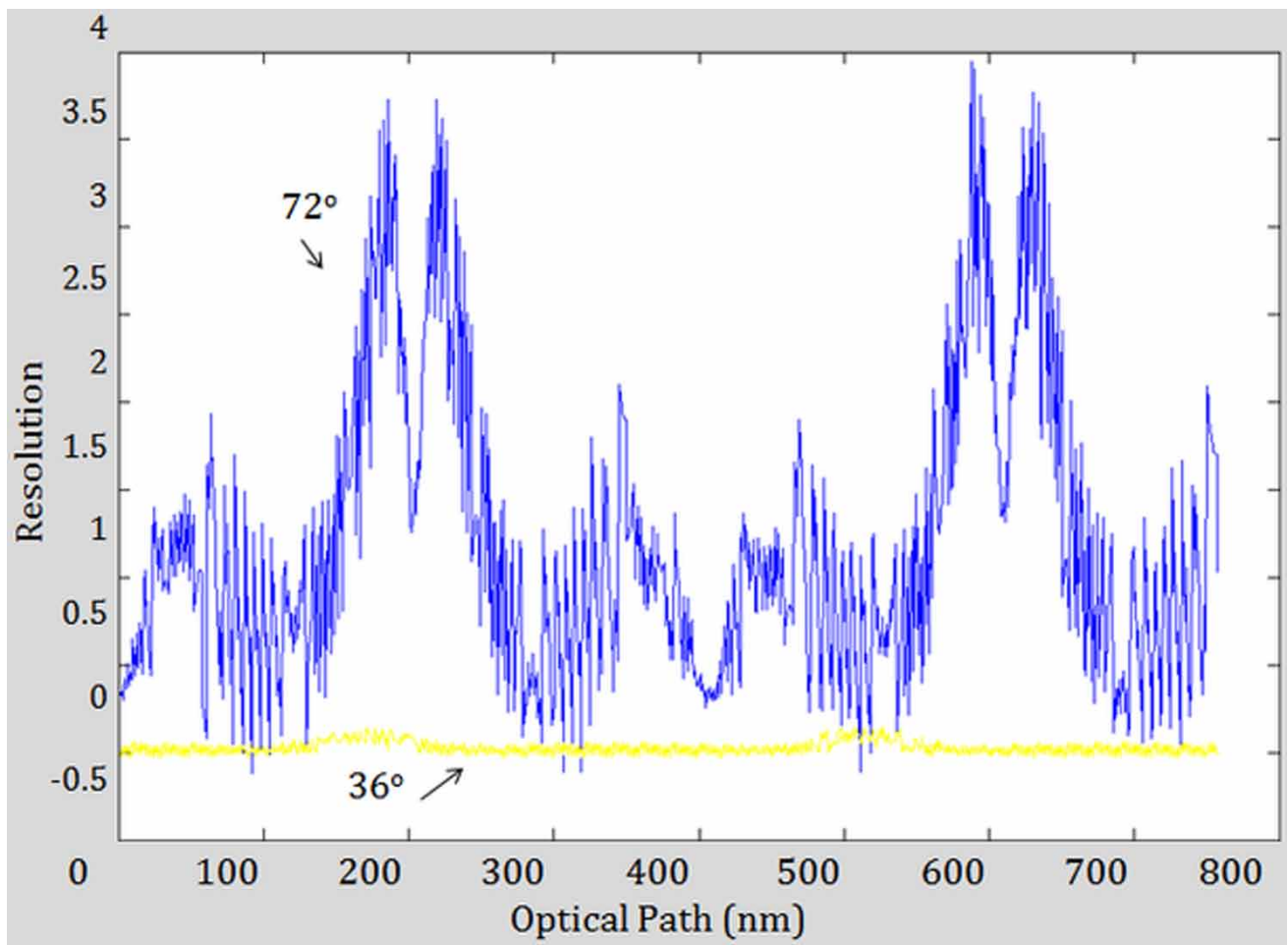

is at least $0.90 \mathrm{~cm}^{2}$. This can be considered as an excellent result since such size is equivalent to chips' size in the industry, so we can expect covering a full die surface thin film characterization.

In order to increase the resolution, we have calculated the maximal resolution obtained when the measurement is performed using two different wavelengths. The results show that, in this case, the resolution increases to the order of the classical ellipsometric resolution.

Moreover, in case we also consider measurements sharing rays' incident angles of $>10^{\circ}$, one can double the surface of the measurement. In spite the fact that the resolution decreases by 40 times for angles of $10^{\circ}$ (for two wavelengths), this technique enables the measurement of surface areas of more than $2 \mathrm{~cm}^{2}$ and can be useful for many applications

\subsection{Matlab Integrated Functions Running the Whole System}

One of main advantages of this new system, presented in Figure 2, is the fact that the platform is fully controlled by a Matlab complex developed code, made of several functions, shortly described below: 
Figure 8. Scanning results of the imaging system for the FFIE platform. The dot line indicates the $>360$ angle results.

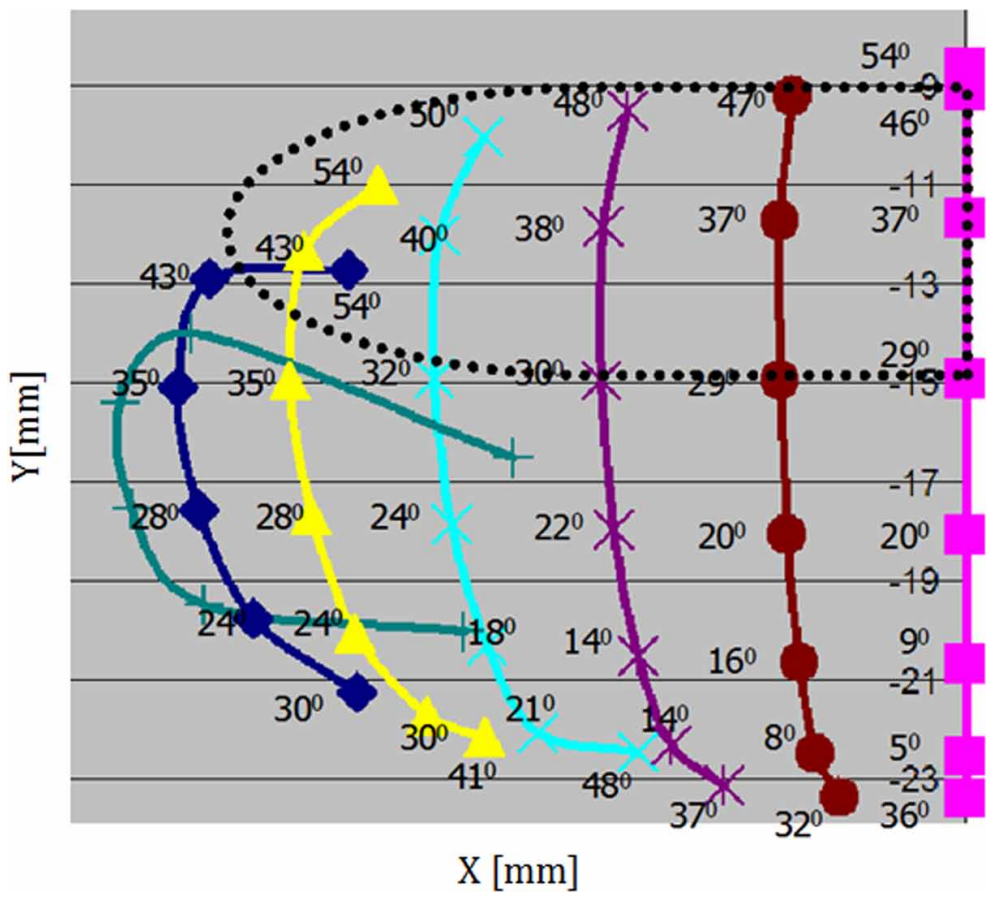

- Ref-Ind: Since the ellipsometer system has been checked for several wavelengths, there is some importance to know the corresponding refractive indexes. As a consequence there is a mathematical need to set a function $n(\lambda)$. Several models exist. We have used the Sellemier function;

- Sfvag_material: The purpose of this function is to provide $\rho_{p}$ and $\rho_{s}$ which represent the reflection coefficients of the surface for the $S$ and $P$ polarizations;

- sfvag_resolution: This function is the heart of the platform and it calculates the resolution of the ellipsometry measurement for a defined thickness;

- sfvag_software: Dedicated to calculating the ratio between the ellipsometer resolution for two different measurement angles. The calculation is done through a loop checking the different layer thicknesses, and providing the measurement resolution for each thickness and the two angles.

\section{CONCLUSION}

A new approach for performing full field imaging ellipsometry is presented and its resolution is compared to the existing techniques. A full complex simulation program has been programed in order to investigate the new approach. The program interacts with all components needed for ellipsometric measurements, and also runs the Oslo 
6 optical design software (Lambda, 2017) to simulate a full imaging ellipsometric optical system.

This study has investigated the influence of different illumination rays' angles of incidence on the resolution, and shown that even small angle deviations from the customary angle cause a major resolution decrease. This investigation also shows that the resolution of the measurements is thickness dependent and is periodic as function of the thickness of the coating layer. Using typical optical imaging system; areas of up to 0.9 square centimeters are illuminated by rays of light having incident angles exceeding $36^{\circ}$, and thus, can be measured simultaneously with acceptable resolution. However, measuring using at least two different wavelengths the resolution may be increased to the order of the classical ellipsometric resolution.

There are, at least, three important novelties presented here:

- The approach performs simultaneous 2D measurements of a thin layered object, and enables the integration of all the mapped spots in order to obtain a full $2 \mathrm{D}$ ellipsometric measurement of the object;

- A special built program interacts with all components needed for ellipsometric measurements, and also runs the Oslo 6 optical design software to simulate a full imaging ellipsometric optical system;

- This simple method enables areas of up to $2.0 \mathrm{~cm}^{2}$ to be measured with high accuracy.

As a preliminary conclusion, our approach can be considered as a good result since such area size is equivalent to the size of chips in the industry, so one can expect covering a full die surface thin film characterization with acceptable resolution. The next step would consist in applying the method in real testing in order to check the solidity and simplicity of the approach.

\section{ACKNOWLEDGMENT}

This research received no specific grant from any funding agency in the public, commercial, or not-for-profit sectors. 


\section{REFERENCES}

Acurion Scientific Instruments. (2004). Imaging Ellipsometry: The next step forward. Nanofilm surface analysis white paper. Menlo Park, USA: Accurion.

Arieli, Y., Wolfling, S., Lanzmann, E., Feigin, G., Kuzniz, T., \& Saban, Y. (2012). U.S. Patent No. 8,319,975. Washington, DC: U.S. Patent and Trademark Office.

Arwin, H. (2001). Is ellipsometry suitable for sensor applications? Sensors and Actuators. A, Physical, 92(1), 43-51. doi:10.1016/S0924-4247(01)00538-6

Asinovski, L., Beaglehole, D., \& Clarkson, M. T. (2008). Imaging ellipsometry: Quantitative analysis. Physica Status Solidi. A, Applications and Materials Science, 205(4), 764-771. doi:10.1002/pssa.200777855

Born, M., \& Wolf, E. (2013). Principles of optics: Electromagnetic theory of propagation, interference and diffraction of light. Elsevier.

Han, C. Y., \& Chao, Y. F. (2006). Photoelastic modulated imaging ellipsometry by stroboscopic illumination technique. The Review of Scientific Instruments, 77(2), 023107. doi:10.1063/1.2173027

Ho, J. H., Lee, C. L., \& Lei, T. F. (1990). Ellipsometry measurements on refractive index profiles of thin films. IEEE Transactions on Instrumentation and Measurement, 39(4), 642-648. doi:10.1109/19.57248

Jin, G. (2008). Development of biosensor based on imaging ellipsometry. Physica Status Solidi. A, Applications and Materials Science, 205(4), 810-816. doi:10.1002/ pssa.200777810

Jin, G., Meng, Y. H., Liu, L., Niu, Y., Chen, S., Cai, Q., \& Jiang, T. J. (2011). Development of biosensor based on imaging ellipsometry and biomedical applications. Thin Solid Films, 519(9), 2750-2757. doi:10.1016/j.tsf.2010.12.175

Jin, G., Tengvall, P., Lundström, I., \& Arwin, H. (1995). A biosensor concept based on imaging ellipsometry for visualization of biomolecular interactions. Analytical Biochemistry, 232(1), 69-72. doi:10.1006/abio.1995.9959 PMID:8600834

Jung, I., Vaupel, M., Pelton, M., Piner, R., Dikin, D. A., Stankovich, S., \& Ruoff, R. S. et al. (2008). Characterization of thermally reduced graphene oxide by imaging ellipsometry. The Journal of Physical Chemistry C, 112(23), 8499-8506. doi:10.1021/ jp802173m

Karageorgiev, P., Orendi, H., Stiller, B., \& Brehmer, L. (2001). Scanning near-field ellipsometric microscope-imaging ellipsometry with a lateral resolution in nanometer range. Applied Physics Letters, 79(11), 1730-1732. doi:10.1063/1.1403237

Lambda Research Corporation. (2017). Oslo professional software. Retrieved from http://oslo-edu.software.informer.com/6.6/ 
Matsubara, M. (1977). U.S. Patent No. 4,037,934. Washington, DC: U.S. Patent and Trademark Office.

Müller, U. R. (2005). In D. V. Nicolau (Ed.), Microarray technology and its applications (pp. 73-77). Berlin: Springer. doi:10.1007/3-540-26578-3_5

Sagnard, F., Bentabet, F., \& Vignat, C. (2005). In situ measurements of the complex permittivity of materials using reflection ellipsometry in the microwave band: Theory (part I). IEEE Transactions on Instrumentation and Measurement, 54(3), 1266-1273. doi:10.1109/TIM.2005.847203

Avi Karsenty received a PhD degree in Applied Physics /Material Science (Microelectronics and Electro-Optics) from the Hebrew University of Jerusalem in 2003. His research focuses on nanoscale electro-optics coupled-devices. After 22 years in High-Tech industries, part of which as Engineer and Manager for 16 years with Intel, he is today the Physics/Electro-Optics Engineering Department's Head. Dr. Karsenty is IEEE Senior Member and OSA Senior Member, and received 38 Awards in Engineering/Physics.

Shmuel Feldman received his BSc degree in Applied Physics / Electro-Optics Engineering Department from the Jerusalem College of Technology in 2008.

Zvi Veig holds a B.Sc degree in Applied Physics/Electro-optic Engineering and a teaching certificate, both from the Jerusalem College of Technology. For the past nine years, Zvi has been employed as an R\&D Engineer in the fields of Electro-optics and Electronics and as a software and hardware team leader. During the past two years Zvi has become a group leader in the area of Unmanned systems. The bulk of his employment experience has been in the area of System Engineering, technological and project management. Zvi is in the process of completing his M.Sc in Bio-medical Engineering at the Technion, Israel Institute of Technology.

Yoel Arieli received his PhD Degree in Applied Physics \& Material Science (Electro-Optics Division) from the Hebrew University of Jerusalem (HUJI), Israel. He is the former Head of the Applied Physics/Electro-Optics Engineering Department at the Lev Academic Center. His expertise is in Spectroscopy, Interferometry, and Imaging, and his interests of research are OCT, Hyperspectral imaging, and Thermography. He has a professional experience of CTO for 15 years and of Consultant for 10 years. 